\title{
Scattering Problem with Physical Behavior of Scattering Matrix and Operator Relations. *
}

\author{
P. Kurasov, \\ Dept. of Math., Stockholm Univ., 10691 Stockholm, SWEDEN; \\ Dept. of Math., Luleå Univ., 97187 Luleå, SWEDEN; \\ Dept. of Math., Ruhr Uni.-Bochum, 44780 Bochum, GERMANY; \\ Dept. of Math. and Comp. Physics, St.Petersburg Univ., \\ 198904 St.Petersburg, RUSSIA; \\ B. Pavlov, \\ The University of Auckland, NEW ZEALAND
}

27 November 1997

\section{Introduction}

It is known that Krein formula for generalized resolvents of selfadjoint extensions of symmetric operators may produce not only the resolvent of an operator, but sometimes the resolvent of an operator relation, which corresponds to the singular choice of the unitary von-Neumann parameter with an eigenvalue equal to one. The formal von-Neumann construction gives an operator relation in this case, since the domain of it is not dense. The denominator of the additional term in Krein formula for generalized resolvents is growing linearly in this case. The remarkable fact is that precisely this property of Krein formula is necessary for the scattering matrix to have physical behavior at infinity

$$
S(k) \longrightarrow 1 \text { when } k \rightarrow \infty,
$$

${ }^{*}$ Published in Operator theory: Advances and Applications, 113 (2000), 195-204. 
(see [4]). In this paper we show that the linear term in the asymtotics of the denominator can appear even if no operator relation occures. Actually the operators (not operator relations) with exactly this property have been studied in a series of papers devoting to the investigation of Schrödinger operators with resonance interaction ("Inner structure") generated by a finite matrix $([1,4,5,7])$.

We study also the following question: Can operator relation occur if one considers generalizations of the resonance interactions involving symmetric operators with arbitrary finite deficiency indices? (All operator considered earlier have essentially deficiency indices $(1,1)$.) It is proven that the operator relations can occur, but are not interesting for applications, since the corresponding generalized resolvents just coincide with the generalized resolvents of the selfadjoint operators appeared by restriction of the operator relations to the subspace of "physically realizable" elements, which belong to the linear hull of elements, generated by the "outer" space. Thus only the operator-part of the relation plays role in scattering processes.

The authors were supported by the Grant number 3368152 from the Marsden Fund of the Royal Society of New Zealand, by the system of Grants ESPRIT21042, CTIAC, awarded by the Commission in Research and Development of European Community, and by the grant number 97-01-01149 of the Russian Fund for Fundamental Research.

\section{Residue of the resolvent at infinity.}

Let us consider first a bounded selfadjoint operator $A$ acting in the Hilbert Space $E$. Assume that $N \subset E, \operatorname{dim} N=n$ is a subspace such that the angle between $N$ and $\hat{N} \equiv \frac{A+i I}{A-i I}$ is positive. This permits developing the construction of extensions, which is alternative to the classical von Neumann construction. This construction uses the symmetric Krein parameter for description of all selfadjoint extensions instead of the unitary parameter in von-Neumann case. This alternative approach provides a straightforward proof of Krein formula, [5]. We are going to explain below some details of this construction.

In what follows the subspaces $N, \hat{N}$ play roles of deficiency subspaces at the points $\lambda=i,-i$ respectively. It is clear, that the Cayley transform of $A$ transfers $E \ominus N$ into $E \ominus \hat{N}$ and $N$ into $\hat{N}$. Redefining the Cayley transform on $N:\left.\left.U\right|_{N} \rightarrow U^{\prime}\right|_{N}$ we get a new unitary operator $U^{\prime}$, which coincides with $U$ on $E \ominus N$. If 1 is not an eigenvalue of it then the inverse Cayley transform 
$A^{\prime}=i \frac{U^{\prime}+1}{U^{\prime}-1}$ exists and is a densely defined selfadjoint operator in E. If 1 is an eigenvalue, then the latter formula defines a selfadjoint operator relation. In both cases the corresponding resolvent is related to the resolvent of the corresponding unitary operator by the formula

$$
R_{\lambda}^{\prime} \equiv\left(A^{\prime}-\lambda 1\right)^{-1}=\frac{1}{i-\lambda} \frac{U^{\prime}-1}{U^{\prime}-\zeta}, \zeta=\frac{\lambda+i}{\lambda-i},
$$

and satisfies the Hilbert relation

$$
R_{\lambda}^{\prime}-R_{\mu}^{\prime}=(\lambda-\mu) R_{\lambda}^{\prime} R_{\mu}^{\prime}
$$

In the singular case the residue of the resolvent at infinity is not equal to identity but the following equality holds

$$
\frac{1}{2 \pi i} \oint R_{\lambda}^{\prime} d \lambda=-I+P_{0}
$$

where $P_{0}$ is the orthogonal projection onto the singular subspace $N_{0} \subset N$ which corresponds to the singular eigenvalues $\zeta=1$ of $U^{\prime}$. Then the restriction of the relation $A^{\prime}$ onto $E \ominus N_{0}$ is a usual selfadjoint operator.

The reduction of an operator relation to the operator can be done for finite rank perturbations of unbounded operators as well with the only difference, that 1 could be not the isolated eigenvalue of the Cayley transform $U^{\prime}$, but an embedded eigenvalue. Nevertheless the splitting of the corresponding singular subspace gives obviously a usual selfadjoint operator in the remaining part of the Hilbert space.

Consider now the case where the nonperturbed operator acts in an orthogonal sum $\mathcal{H}$ of two Hilbert spaces $\mathcal{H}=E \oplus H$ and is equal to the orthogonal sum of two operators $\mathcal{A}=A \oplus L$ acting in the spaces $E$ and $H$ correspondingly. We suppose that the first operator is bounded, the second one is unbounded. In the following section the role of $A$ is played by a finite matrix, and the role of $L$ is played by the Laplacian in $L_{2}\left(\mathbf{R}^{3}\right)$.

Let us asume that $N_{e}$ and $N_{h}$ are finite dimensional deficiency subspaces at point $\lambda=i$ for the restricted operators $A_{0}$ and $L_{0}$ respectively. Then the sum $\mathcal{N}=N_{e} \oplus N_{h}$ is a deficiency subspace for the total restricted operator $\mathcal{A}_{0}=A_{0} \oplus L_{0}$ and $\operatorname{Dom}\left(\mathcal{A}_{0}\right)=\frac{I}{\mathcal{A}-i I}[\mathcal{H} \ominus \mathcal{N}]$. We assume, that $\mathcal{N} \cap \operatorname{Dom}(L)=$ $\emptyset$, so that the domain of the restricted operator $L_{0}$ is dense in $H$.

In what follows we need the expression for singular subspace in terms of Krein parameters. The Krein formula for the singular case has the same form as for non-singular case (see [6],[7],[9]). Denoting by $\Gamma$ any bounded 
selfadjoint operator in $\mathcal{N}$ (Krein parameter) represented by the operatormatrix

$$
\Gamma=\left(\begin{array}{cc}
\gamma_{e e} & \gamma_{e h} \\
\gamma_{h e} & \gamma_{h h}
\end{array}\right)
$$

we write this formula as

$$
\left(\mathcal{A}_{\Gamma}-\lambda I\right)^{-1}=(\mathcal{A}-\lambda I)^{-1}+\frac{\mathcal{A}+i I}{\mathcal{A}-\lambda I} \frac{I}{\Gamma-\mathcal{Q}(\lambda)} P_{\mathcal{N}} \frac{\mathcal{A}-i I}{\mathcal{A}-\lambda I},
$$

where

$$
\mathcal{Q}(\lambda)=\left(\begin{array}{cc}
\left.P_{N_{e}} \frac{I+\lambda A}{A-\lambda I}\right|_{N_{e}} & 0 \\
0 & \left.P_{N_{h}} \frac{I+\lambda L}{L-\lambda I}\right|_{N_{h}}
\end{array}\right) \equiv\left(\begin{array}{cc}
Q_{A}(\lambda) & 0 \\
0 & Q_{L}(\lambda)
\end{array}\right)
$$

and $P_{\mathcal{N}}$ is the orthogonal projector on $\mathcal{N}$. Consider the case where the total operator $\mathcal{A}$ is bounded, i.e. where $\mathcal{A}=A$ and $\Gamma=\gamma_{e e}$. Then the residue of the resolvent at infinity can easily be calculated using the spectral representation of the finite dimensional operator $\Gamma+P_{N} A P_{N}$ acting in $N$ with respect to the weighted scalar product

$$
\ll g, h \gg \equiv<P_{N}\left(I+A^{2}\right) P_{N} g, h>.
$$

Lemma 1 If the total original operator is bounded $\mathcal{A}=A$ then the residue of the resolvent $\left(\mathcal{A}_{\Gamma}-\lambda I\right)^{-1}$ at infinity is equal to $I-P_{0}$, where

$$
P_{0} *=\sum_{\alpha_{s}=0}(A+i I) e_{s}<(A-i I) *, e_{s}>
$$

and $e_{s}$ are the eigenvectors of the operator $\Gamma+\left.P_{N} A\right|_{N}$ corresponding to the eigenvalue $\alpha_{s}$ and orthonormalized with respect to the weighted scalar product $\ll \cdot, \cdot \gg$.

Note that the sum in the latter formula is taken over all eigenvectors corresponding to the zero eigenvalue of the operator $\gamma_{e e}+\left.P_{N_{e}} A\right|_{N_{e}}=\Gamma+\left.P_{\mathcal{N}} \mathcal{A}\right|_{\mathcal{N}}$. Proof. Note that the additional term $P_{0}$ of the residue is determined by the second term in Krein formula

$$
P_{0}=(A+i I) \lim _{\lambda \rightarrow \infty} \frac{1}{\lambda\left[\Gamma-\left.P_{N} \frac{I+\lambda A}{A-\lambda I}\right|_{N}\right]} P_{N_{e}}(A-i I) .
$$

If the operator $A$ is bounded, then the asymptotic representation for the inverse operator in the latter formula for large values of $\lambda$ can be calculated via 
the perturbation procedure. Really, for bounded operator $A$ the denominator for large values of $\lambda$ can be represented by convergent series

$$
\lambda\left[\Gamma+\left.P_{N} A\right|_{N}\right]+\left.P_{N}\left(1+A^{2}\right)\right|_{N}+\sum_{l=1}^{\infty} \frac{\left.P_{N} A^{l}\left(1+A^{2}\right)\right|_{N}}{\lambda^{l}} .
$$

The leading terms of this series can be inverted using the spectral representation of $\Gamma+\left.P_{N} A\right|_{N}$ in $N$ with respect to the weighted scalar product $\ll \cdot, \cdot \gg$ :

$$
\left(\Gamma+P_{N} A\right) e_{s}=\alpha_{s} P_{N}\left(1+A^{2}\right) e_{s} .
$$

Assuming, that the eigenvectors are orthonormalized $\ll e_{s}, e_{t} \gg=\delta_{s t}$, we get for the inverse of the leading pair :

$$
\begin{aligned}
& {\left[\lambda \Gamma+\left.\lambda P_{N} A\right|_{N}+\left.P_{N}\left(1+A^{2}\right)\right|_{N}\right]^{-1} f=} \\
& =\sum_{s} \frac{1}{1+\lambda \alpha_{s}} e_{s}<f, e_{s}>\equiv \mathcal{R}_{0} f
\end{aligned}
$$

The term corresponding to the eigenvalue $\alpha_{s}=0$ is constant, and the remaining terms tend to zero when $\lambda$ goes to infinity. The operator $\mathcal{R}_{0}$ is bounded. Taking into account, that the sum of the remaining terms of the series is small for lagre $\lambda$

$$
\sum_{l=1}^{\infty}\left\|\frac{\left.P_{N} A^{l}\left(1+A^{2}\right)\right|_{N}}{\lambda^{l}}\right\|=O\left(\frac{1}{\lambda}\right),
$$

we conclude, that

$$
\frac{1}{\lambda\left[\Gamma-\left.P_{N} \frac{I+\lambda A}{A-\lambda I}\right|_{N}\right]}=\mathcal{R}_{0}\left[1+O\left(\frac{1}{\lambda}\right) \mathcal{R}_{0}\right]^{-1},
$$

and hence

$$
\begin{aligned}
P_{0} f & =(A+i I) \lim _{\lambda \rightarrow \infty} \frac{1}{\lambda\left[\Gamma-\left.P_{N} \frac{I+\lambda A}{A-\lambda I}\right|_{N}\right]} P_{N_{e}}(A-i I) f \\
& =\lim _{\lambda \rightarrow \infty}(A+i I) \mathcal{R}_{0} P_{N_{e}}(A-i I) f \\
& =\sum_{\alpha_{s}=0}(A+i I) e_{s}<f,(A+i I) e_{s}>
\end{aligned}
$$

Notice, that the dot product in the latter formula is not weighted, hence the operator $P_{0}$ is an orthogonal projection in $E$ onto $(A+i I) N_{0}$. The Lemma is proven. 
In the case where the nontrivial unbounded component is present, the extensions could be parametrised by the boundary conditions imposed onto abstract boundary values $\xi_{ \pm}$of the elements ( see [7]) :

$$
\begin{array}{r}
u_{N_{e}} \sim \frac{A}{A-i I} \xi_{+}^{e}+\frac{1}{A-i I} \xi_{-}^{e} \in N_{e}+\hat{N}_{e} \\
h=h_{0}+\frac{L}{L-i I} \xi_{+}^{h}+\frac{1}{L-i I} \xi_{-}^{h} \in H, \\
\left(\begin{array}{c}
\xi_{-}^{e} \\
\xi_{-}^{h}
\end{array}\right)=\left(\begin{array}{cc}
\gamma_{e e} & \gamma_{e h} \\
\gamma_{h e} & \gamma_{h h}
\end{array}\right)\left(\begin{array}{c}
\xi_{+}^{e} \\
\xi_{+}^{h}
\end{array}\right) .
\end{array}
$$

Straightforward calculation gives the two-channel Krein formula

$$
\begin{aligned}
& \left(\mathcal{A}_{\Gamma}-\lambda I\right)^{-1}=\left(\begin{array}{cc}
\frac{I}{A-\lambda I} & 0 \\
0 & \frac{I}{L-\lambda I}
\end{array}\right)+ \\
& \left(\begin{array}{cc}
\frac{A+i I}{A-\lambda I} & 0 \\
0 & \frac{L+i I}{L-\lambda I}
\end{array}\right)\left(\begin{array}{cc}
\gamma_{e e}-\left.P_{N_{e}} \frac{I+\lambda A}{A-\lambda I}\right|_{N_{e}} & \gamma_{e h} \\
\gamma_{h e} & \gamma_{h h}-\left.P_{N_{h}} \frac{I+\lambda L}{L-\lambda I}\right|_{N_{h}}
\end{array}\right)^{-1} P_{\mathcal{N}} \\
& \left(\begin{array}{cc}
\frac{A+i I}{A-\lambda I} & 0 \\
0 & \frac{L+i I}{L-\lambda I}
\end{array}\right) .
\end{aligned}
$$

Calculation of the residue of the resolvent at infinity in this case obviously requires comparison of the behavior of $Q$-operators $Q_{A}(\lambda)$ and $Q_{L}(\lambda)$ at infinity. Therefore we are going to study in the following section the particular case, where the comparison can be easily carried out.

General scattering problem can be studied using technique developed in [1]. The case where the deficiency indices of the operator $L_{0}$ are equal to $(1,1)$ has been investigated in detail $[2,8]$. Physically relevant are the restrictions of the resolvent of the total operator $\mathcal{A}_{\Gamma}$ to the space $H$. These restrictions coincide with the generalized resolvents corresponding to the operator $L_{0}$. It has been proven that one can obtain arbitrary generalized resolvent considering only the restrictions of the operator $A$ with the deficiency indices $(1,1)$. Moreover it has been proven that if both restricted operators $A_{0}$ and $L_{0}$ have deficiency indices $(1,1)$, the boundary operator $\Gamma$ is not singular $\left(\gamma_{e h} \neq 0 \neq \gamma_{h e}\right)$, then $\mathcal{A}_{\Gamma}$ is a selfadjoint operator (not an operator relation). In the exceptional case, where $\gamma_{e h}=\gamma_{h e}=0, \mathcal{A}_{\Gamma}$ is equal to the orthogonal sum of a selfadjoint operator acting in $H$ and may be an operator relation in $E$. The latter case is not interesting, since the generalized resolvent in this 
case coincides with the resolvent of a selfadjoint operator acting in $H$. In the following section we show how to generalize the resolvent analysis to the case of unbounded operator.

\section{Resolvent analysis of a soluble model.}

Let us consider the selfadjoint operator $\mathcal{A}=A \oplus(-\triangle)$ acting in the orthogonal sum $E \oplus L_{2}\left(\mathbf{R}^{3}\right)$ with the finitedimensional component $E$. Reducing the Laplacian to the domain containing all smooth square integrable functions vanishing at the origin we get the adjoint operator $-\Delta^{+}$defined on locally $W_{2}^{2}\left(\mathbf{R}^{3} \backslash\{0\}\right)$ functions outside the origin with the asymptotic behavior

$$
u(x)=\frac{a}{4 \pi|x|}+b+o(1), x \rightarrow 0 .
$$

The constants $a=a_{u}, b=b_{u}$ play the role of boundary data of the element $u$ at the origin and the corresponding boundary form is equal to

$$
<-\triangle^{+} u, v>-<u,-\triangle^{+} v>=b_{u} \bar{a}_{v}-a_{u} \bar{b}_{v} .
$$

The boundary data and the boundary form are nontrivial only on the sum of deficiency subspaces of $L_{0}$ :

$$
M_{h}=N_{h}+\hat{N}_{h}=\bigvee\left(\frac{e^{i k|x|}}{4 \pi|x|}, \frac{e^{-i \bar{k}|x|}}{4 \pi|x|}\right), k^{2}=i
$$

The finitedimensional operator $A$ is restricted $A \rightarrow A_{0}$ onto domain $\operatorname{Dom}\left(A_{0}\right)=$ $\frac{1}{A-i I}(E \ominus N), N$ playing the role of the deficiency subspace at the spectral point $i, N=E \ominus\left(A_{0}-i I\right) \operatorname{Dom}\left(A_{0}\right)$. If the angle between $N_{e}$ and $\hat{N}_{e}$ is positive, then the elements $\nu$ from the orthogonal sum $M_{e}=N_{e}+\hat{N}_{e}$ can be characterized by the boundary values $\xi_{ \pm}$:

$$
\nu=\frac{A}{A-i I} \xi_{+}^{\nu}+\frac{1}{A-i I} \xi_{-}^{\nu},
$$

and the formal adjoint $A_{0}^{+}$defined on $M_{e}$ by the formulas

$$
\begin{aligned}
A_{0}^{+}\left(\frac{A}{A-i I} \xi_{+}^{\nu}\right) & =-\frac{1}{A-i I} \xi_{+}^{\nu} \\
A_{0}^{+}\left(\frac{1}{A-i I} \xi_{-}^{\nu}\right) & =\frac{A}{A-i I} \xi_{-}^{\nu}
\end{aligned}
$$


has the boundary form

$$
<A_{0}^{+} \nu, \mu>_{E}-<\nu, A_{0}^{+} \mu>_{E}=<\xi_{-}^{\nu}, \xi_{+}^{\mu}>-<\xi_{+}^{\nu}, \xi_{-}^{\mu}>
$$

The latter boundary form can be extended to the formal sum Dom $\left(A_{0}\right)+M_{e}$.

The selfadjoint operator extensions and operator relations of the symmetric operator $A_{0} \oplus L_{0}$ are parametrised by Lagrangian planes $\mathcal{L}$ of the total boundary form in $M_{e} \oplus M_{h}$, see [5]. In particular these planes $\mathcal{L}_{\Gamma}$ can be described by the boundary conditions defined by the bounded selfadjoint operators $\Gamma$ connecting the boundary data:

$$
\left(\begin{array}{cc}
\gamma_{e e} & \gamma_{e h} \\
\gamma_{h e} & \gamma_{h h}
\end{array}\right)\left(\begin{array}{c}
\xi_{+} \\
a
\end{array}\right)=\left(\begin{array}{c}
\xi_{-} \\
b
\end{array}\right)
$$

similarly to [3]. One can easily check, that the operator $\left.\mathcal{A}\right|_{\mathcal{L}}$ defined on the Lagrangian plane $\mathcal{L}$ as a sum of adjoint $L_{0}^{+}$and the formal adjoint $A_{0}^{+}$ is symmetric on $\mathcal{L}$. Attaching to it the operators $A_{0}$ and $L_{0}$ we get the selfadjoint operator or operator relation in $E \oplus L_{2}\left(R_{3}\right)$.

Theorem 2 The sum $\mathcal{D}_{\Gamma}=D\left(A_{0}\right)+\mathcal{L}+D\left(L_{0}\right)$ is a direct one in $E \oplus L_{2}\left(\mathbf{R}^{3}\right)$ and represents the domain of a selfadjoint operator or operator relation $\mathcal{A}_{\Gamma}$ defined by the corresponding Krein formula for the resolvent

$$
\begin{aligned}
& \left(\mathcal{A}_{\Gamma}-\lambda I\right)^{-1}=\left(\begin{array}{cc}
\frac{I}{A-\lambda I} & 0 \\
0 & \int_{\mathbf{R}^{3}} \frac{e^{i k|x-y|}}{4 \pi|x-y|} \cdot d^{3} y
\end{array}\right)+ \\
& \left(\begin{array}{cc}
\frac{A+i I}{A-\lambda I} & 0 \\
0 & \frac{e^{i k|x|}}{4 \pi|x|}
\end{array}\right)\left(\begin{array}{cc}
\gamma_{e e}-P_{N}\left(\frac{I+\lambda A}{A-\lambda I}\right) & \gamma_{e h} \\
\gamma_{h e} & \gamma_{h h}-\frac{i k}{4 \pi}
\end{array}\right)^{-1}\left(\begin{array}{cc}
P_{N_{e}} \frac{A-i I}{A-\lambda I} & 0 \\
0 & \int_{\mathbf{R}^{3}} \frac{e^{i k|y|}}{4 \pi|y|} \cdot d^{3} y
\end{array}\right),
\end{aligned}
$$

where $k^{2}=\lambda$.

The proof of this statement can be achieved by minor modification of one of standard proofs of Krein formula taking into account the fact, that the restricted operator is not densely defined ( see [3],[5],[9]).

Our aim is investigation of asymptotic behavior of the resolvent at infinity and calculation of the corresponding residue. The central part in the calculation of the resolvent is the calculation of the inverse matrix in the second term of the expression for the generalized resolvent. 
Lemma 3 Let us denote by $K$ the expression

$$
K \equiv \gamma_{h h}-\frac{i k}{4 \pi}-\gamma_{h e} \frac{I}{\gamma_{e e}-Q_{0}} \gamma_{e h}
$$

Then the following representation of the inverse matrix is true:

$$
\begin{gathered}
\left(\begin{array}{cc}
\gamma_{e e}-P_{N}\left(\frac{I+\lambda A}{A-\lambda I}\right) & \gamma_{e h} \\
\gamma_{h e} & \gamma_{h h}-\frac{i k}{4 \pi}
\end{array}\right)^{-1}= \\
\left(\begin{array}{cc}
\frac{1}{\gamma_{e e}-Q_{0}}\left[1+\gamma_{e h} \frac{1}{\gamma_{e e}-Q_{0}} \gamma_{h e} \frac{1}{K} \frac{1}{\gamma_{e e}-Q_{0}}\right] & -\frac{1}{\gamma_{e e}-Q_{0}} \gamma_{e h} \frac{1}{K} \\
-\frac{1}{K} \gamma_{h e} \frac{1}{\gamma_{e e}-Q_{0}} & \frac{1}{K}
\end{array}\right)
\end{gathered}
$$

Proof is obtained by straightforward calculation.

Now we calculate the residue of the resolvent at infinity separately in "internal" space $E$ and in "external" space $L_{2}\left(\mathbf{R}^{3}\right)$.

Let us consider basis $e_{s}$ in $N$ introduced in the previous section. The vector $\gamma=\sum_{\alpha_{s}=0}(A+i I) e_{s}<\gamma_{e h}, e_{s}>\in N_{0}$ is actually the orthogonal projection of $(A+i I)^{-1} \gamma_{e h}$ onto $M_{0}=(A+i I) N_{0}$. Denoting by $\gamma_{0}$ the corresponding normalized vector $\gamma_{0}=\frac{\gamma}{|\gamma|}$ we form the orthogonal projection in $E$ onto $M_{\gamma} \equiv M_{0} \ominus\{\gamma\}$ as

$$
P_{M} *-\gamma_{0}<*, \gamma_{0}>\equiv P_{M_{\gamma}} * .
$$

Theorem 4 The residue of the resolvent of the total operator $\mathcal{A}_{\Gamma}$ at infinity is equal to $I-P_{M_{\gamma}}$ where $P_{M_{\gamma}}$ is the orthogonal projector onto $M_{\gamma}$ in $E \oplus$ $L_{2}\left(\mathbf{R}^{3}\right)$.

Proof. Straightforward calculation of the expression $K$ for large values of $\lambda$ gives

$$
\begin{aligned}
K & \approx \gamma_{h h}-\frac{i k}{4 \pi}-\sum_{s} \frac{\lambda}{1-\lambda \alpha_{s}}\left|<\gamma_{e h}, e_{s}>\right|^{2} \\
& =-\lambda \sum_{\alpha_{s}=0}\left|<\gamma_{e h}, e_{s}>\right|^{2}-\frac{i k}{4 \pi}+\gamma_{h h}-\lambda \sum_{l \geq 1} \sum_{\alpha_{s} \neq 0} \frac{\left|<\gamma_{e h}, e_{s}>\right|^{2}}{\left(-\alpha_{s} \lambda\right)^{l}} \\
& =-\lambda \sum_{\alpha_{s}=0}\left|<\gamma_{e h}, e_{s}>\right|^{2}+O(1) .
\end{aligned}
$$


Then the integration of the ee-element of the resolvent over the large circle gives precisely $I-P_{M_{\gamma}}$. The integration of the remaining elements of the resolvent applied to the dense in $L_{2}\left(\mathbf{R}^{3}\right)$ lineal of all functions $g_{1}$, for which $\int \frac{g_{1}(x)}{|x|} d x=0$ gives no contribution, since each of the elements contains the factor $\frac{e^{i k|x|}}{4 \pi|x|}$, for instance the $e h$-element of the kernel of the additional term in Krein formula gives the residue:

$$
\frac{\sum_{\alpha_{s}=0}(A+i I) e_{s}<\gamma_{e h}, e_{s}>}{\sum_{\alpha_{s}=0}\left|<\gamma_{e h}, e_{s}>\right|^{2}} \frac{1}{4 \pi|x|},
$$

which vanishes on this dense lineal. The theorem is proven.

At the same time the straightforward calculation shows, that any element $\nu$ from the subspace $M_{\gamma}$

$$
\nu=\left(\begin{array}{c}
(A+i I) e \\
0
\end{array}\right), \text { where } e \in N_{e},\left[\gamma_{e e}+\left.P_{N} A\right|_{N}\right] e=0,<\gamma_{e h}, e>=0
$$

satisfies the equation $\left(\mathcal{A}_{\gamma}-\lambda I\right)^{-1} \nu=0$ for each $\lambda$. Really

$$
\begin{array}{ccc}
P_{N_{e}} \frac{A-i}{A-\lambda I}(A+i I) e & = & P_{N_{e}} \frac{A^{2}+1}{A-\lambda} \\
P_{N_{e}} A e+P_{N_{e}} \frac{I+\lambda A}{A-\lambda I} e & = & -\gamma_{e e} e+Q_{0}(\lambda) e .
\end{array}=
$$

On the other hand

$$
\left(\begin{array}{cc}
\gamma_{e e}-P_{N_{e}}\left(\frac{I+\lambda A}{A-\lambda I}\right) & \gamma_{e h} \\
\gamma_{h e} & \gamma_{h h}-\frac{i k}{4 \pi}
\end{array}\right)\left(\begin{array}{l}
e \\
0
\end{array}\right)=\left(\begin{array}{c}
\left(\gamma_{e e}-P_{N_{e}}\left(\frac{I+\lambda A}{A-\lambda I}\right) \gamma_{e h}\right) e \\
0
\end{array}\right),
$$

since $\gamma_{h e} e=<e, \gamma_{e h}>=0$. Then we have:

$$
\left(\mathcal{A}_{\gamma}-\lambda I\right)^{-1} \nu=\left(\frac{A+i I}{A-\lambda I} e\right)-\left(\frac{A+i I}{A-\lambda I} e\right)=0 .
$$

It follows that the space $M_{\gamma}$ is contained in the kernel of the resolvent of $\mathcal{A}_{\Gamma}$.

In the case where the deficiency subspace $N_{e}$ has dimension one and the parameters $\gamma_{e h}, \gamma_{h e}$ are not equal to zero, the subspace $M_{\gamma}$ is trivial. It follows that in this case no operator relation occures. If the dimension of the deficiency subspace $N_{e}$ is greater than one, then $\mathcal{A}_{\Gamma}$ can be an operator relation. But the restriction of the operator relation to the orthogonal complement of $M_{\gamma}$ in $E \oplus L_{2}\left(\mathbf{R}^{3}\right)$ is a selfadjoint operator. The resolvent of this new operator restricted to the space $L_{2}\left(\mathbf{R}^{3}\right)$ coincides with the restricted resolvent of the operator relation. Therefore general consideration can be confined to the case where no operator relation occures, since only the generalized resolvent is interesting in applications. 


\section{Scattering Matrix.}

In our case the scattering amplitude is nontrivial in spherically - symmetric channel only. The corresponding scattering amplitude can be found from the condition, that the ansatz

$$
\psi=\left(\begin{array}{c}
\frac{A+i I}{A-\lambda I} \xi_{+} \\
e^{i k<x, \omega>}+f(k) \frac{e^{i k|x|}}{4 \pi|x|}
\end{array}\right)
$$

satisfies the homogeneous equation $\left(\mathcal{A}_{\gamma}-\lambda I\right) \psi=0$ in the distributional sense and the boundary conditions. This gives the following expressions for the scattering amplitude $f$ and the boundary data $\xi_{+}$in the internal channel:

$$
\begin{aligned}
& f(k)=\frac{1}{-\gamma_{h h}+\gamma_{h e}\left(\gamma_{e e}-Q_{A}(\lambda)\right)^{-1} \gamma_{e h}+\frac{i k}{4 \pi}} \\
& \xi_{+}=-\left(\gamma_{e e}-Q_{A}(\lambda)\right)^{-1} \gamma_{e h} f(k) .
\end{aligned}
$$

It is obvious, that under the condition that the orthogonal projection of $(A+i I)^{-1} \gamma_{e h}$ onto $M_{0}=(A+i I) N_{0}$ is not zero, that is the vector $\gamma_{e h}$ is not orthogonal to the null-space of the operator $\gamma_{e e}+\left.P_{N} A\right|_{A}$, the scattering matrix in spherically - symmetric channel

$$
S(k)=\frac{-\gamma_{h h}+\gamma_{h e}\left(\gamma_{e e}-Q_{A}(\lambda)\right)^{-1} \gamma_{e h}-\frac{i k}{4 \pi}}{-\gamma_{h h}+\gamma_{h e}\left(\gamma_{e e}-Q_{A}(\lambda)\right)^{-1} \gamma_{e h}+\frac{i k}{4 \pi}}
$$

tends to 1 as $k^{2}=\lambda \rightarrow \infty$. One can check by direct calculation, that the inner components of scattered waves are orthogonal to the singular subspace of the operator relation.

We have shown that all model operators cosidered in the papers $[1,4,5,7]$ are really selfadjoint operators. These operators can model physically interesting problems only if the denominator in Krein formula for the generalized resolvents has linear asymtotics at infinity. This situation has been usually related with the fact that the Q-function of the operator contains linear term and therefore with the operator relations. We have proven in this paper that this relation is not inevitable.

The authors are grateful to Professor Malamud for fruitful discussions.

\section{References}

[1] V. Adamjan, B. Pavlov, Zero- radius potentials and M.G.Krein formula for generalized resolvents (in Russian), Zap. Nauch. Sem. Leningrad. 
Otdel. Mat. Inst. Steklov. (LOMI), 149 (1986), Issled. Linein. Teor. Functsii. XV, 7-23.

[2] S. Albeverio, P. Kurasov, "Solvable Schrödinger type operators. Singular perturbations of differential operators", in preparation.

[3] V. I. Gorbachuk, "Boundary problems for differential-operator equations", (in Russian) Kiev: Hauk. dumka, 1984, - 284 p.

[4] B. S. Pavlov, Boundary values on thin manifolds and the semiboundedness of the three-body Schrödinger operator with point potential, Math. USSR - Sbornik 64 (1989), 161 -175.

[5] B. S. Pavlov, The theory of the extensions and explicitly-soluble models, Russian Mathematical Surveys 42 (1987), 127 -168.

[6] M. A. Krasnoselskij, On selfadjoint extensions of Hermitian Operators, (in Russian), Ukr.Mat.Journal 1 (1949), 21-38

[7] B. Pavlov, A model of zero-radius potential with internal structure (in Russian), Teor. Mat. Fiz. 59 (1984), 345-353. (English translation: Theoret. and Math. Phys. 59 (1984), 544-550).

[8] P. Kurasov, B. Pavlov, in preparation, to be published in Proc. Conf. "Hyperfunctions, Operator and Dynamical Systems", Brussel, 1997.

[9] M. M. Malamud, On formula of the generalized resolvents of a nondensely defined hermitian operators.(in Russian), Ukr.Mat.Journal. 44 (1992), 1658-1688. (English translation: Sov.Math., Plenum Publ.Corp., 0041-5995/92/4412-1523, 1993, 1522 - 1546) 